\title{
Das Potential eines homogenen rechtwinkligen Parallelepipedums.
}

(Von Hern o. Röthig.)

Der vorliegende Aufsatz hat den Zweck, zu zeigen, dafs das Potential eines homogenen rechtwinkligen Parallelepipedums in Beziehung auf einen beliebigen Punkt durch einen Ausdruck dargestellt wird, welcher auf rationale Weise aus Logarithmen und Arctang. zusammengesetzt ist. Der Beweis dieser Behauptung wird nicht nur direct durch Integration geleistet werden, sondern auch durch Verification des gefundenen Ausdruckes nach der von Dirichlet im $32^{\text {sten }}$ Bande dieses Journals gegebenen allgemeinen Methode.

Nennt man die Seiten eines rechtwinkligen Parallelepipedums $2 a, 2 b, 2 c$ und legt den Anfangspunkt der Coordinaten in den Mittelpunkt, so ist das zu bestimmende Potential in Beziehung auf einen Punkt $(\xi, \eta, \zeta)$ ausgedrückt durch:

wo

$$
\text { (1.) } \boldsymbol{P}=\int_{-a}^{+a} \int_{-b}^{+b} \int_{-c}^{+c} \frac{d x d y d z}{r} \text {, }
$$

$$
r^{2}=(x-\xi)^{2}+(y-\eta)^{2}+(z-\zeta)^{2}
$$

und die constante Dichtigkeit gleich der Einheit gesetzt ist. In dieser Gleichung sind die Gröfsen $a, b, c$ ihrer Bedeutung nach positiv, es soll deshalb der von dieser Einschränkung unabhängige Werth des Integrales in (1.) mit $\Phi(a, b, c, \xi, \eta, \zeta)$ bezeichnet werden.

Schreibt man nun in dem Integrale in Bezug auf $x$ in (1.) $x$ für $x-\xi$, so geht dasselbe über in:

wo

$$
\int_{-a-\xi}^{+a-\xi} \frac{d x}{r}=\int_{-a-\xi}^{00} \frac{d x}{r}+\int_{1}^{a-\xi} \frac{d x}{r}
$$

$$
\boldsymbol{r}^{2}=\boldsymbol{x}^{2}+(\boldsymbol{y}-\eta)^{2}+(z-\zeta)^{2} .
$$

Für die beiden Integrale auf der rechten Seite darf aber, wie leicht erhellt, geschrieben werden:

$$
\frac{1}{2} \int_{-(a+\xi)}^{+(a+\xi)} \frac{d x}{r}+\frac{1}{2} \int_{-(a-\xi)}^{+(a-\xi)} \frac{d x}{r},
$$

und durch Einführung dieser Summe für das Integral in Beziehung auf $x$ in (1.) folgt:

(2.) $2 \boldsymbol{P}=\Sigma \Phi(a+\varepsilon \xi, b, c, 0, \eta, \zeta)$, 
wo für $\varepsilon$ die Werthe +1 und -1 zu setzen sind, und die Summe sich auf die Addition der so erhaltenen Glieder bezieht. Behandelt man jetzt auf dieselbe Weise das Integral in Beziehung auf $y$ in dem Ausdruck unter dem Summenzeichen in (2.), so überzeugt man sich leicht, dafs:

$$
\Phi(a+\varepsilon \xi, b, c, 0, \eta, \zeta)=\Sigma \Phi\left(a+\varepsilon \xi, b+\varepsilon^{\prime} \eta, c, 0,0, \zeta\right)
$$

und endlich folgt durch gleiche Behandlung des Integrales in Beziehung auf $z$ :

$$
\Phi\left(a+\varepsilon \xi, b+\varepsilon^{\prime} \eta, c, 0,0, \zeta\right)=\Sigma \Phi\left(a+\varepsilon \xi, b+\varepsilon^{\prime} \eta, c+\varepsilon^{\prime \prime} \zeta, 0,0,0\right) .
$$

Die Summen in beiden Gleichungen beziehen sich resp. auf $\varepsilon^{\prime}$ und $\varepsilon^{\prime \prime}$, welche Gröfsen dieselbe Bedeutung haben wie $\varepsilon$ in (2.).

Durch successive Anwendung der beiden vorstehenden Gleichungen erhält man nun aus (2.), wenn noch in $\Phi$ die Argumente, welche den Werth 0 haben, fortgelassen werden:

$$
\text { (3.) } \quad 8 \boldsymbol{P}=\Sigma \boldsymbol{\Phi}\left(\boldsymbol{a}+\varepsilon \xi, \boldsymbol{b}+\varepsilon^{\prime} \eta, c+\varepsilon^{\prime \prime} \zeta\right) \text {. }
$$

Hierin haben die Gröfsen $\varepsilon, \varepsilon^{\prime}, \varepsilon^{\prime \prime}$ die Werthe +1 und -1 , und die Summe erstreckt sich auf die acht Glieder, welche man dadurch erhält, dafs den Gröfsen $\varepsilon, \varepsilon^{\prime}, \varepsilon^{\prime \prime}$ ihre verschiedenen Werthe beigelegt werden.

Es kommt daher nur noch darauf an einen Ausdruck für $\boldsymbol{\Phi}(\alpha, \beta, \gamma)$, wo $\alpha, \beta, \gamma$ irgend welche reelle Gröfsen bedeuten, zu finden. Nun hat aber zu Folge von (1.) $\Phi(\alpha, \beta, \gamma)$ die Form:

$$
\Phi(\alpha, \beta, \gamma)=\int_{-\alpha}^{+\alpha} \int_{-\beta}^{+\beta} \int_{-\gamma}^{+\gamma} \frac{d x d y d z}{r}
$$

wo:

$$
\boldsymbol{r}^{2}=x^{2}+y^{2}+z^{2} \text {. }
$$

Setzt man hierin $\vartheta \alpha$ für $\alpha, \vartheta \beta$ für $\beta, \vartheta \gamma$ für $\gamma$, so wird:

$$
\boldsymbol{\Phi}(\vartheta \alpha, \vartheta \beta, \vartheta \gamma)=\int_{-\vartheta \alpha}^{+\vartheta \alpha} \int_{-\vartheta \beta}^{+\vartheta \beta} \int_{-\vartheta \gamma}^{+\vartheta \gamma} \frac{d x d y d z}{r},
$$

und wenn nun in diesem Integrale $\vartheta x$ für $x, 9 y$ für $y, \mathscr{g}$ für $z$ geschrieben wird, so folgt:

oder

$$
\Phi(\vartheta \alpha, \vartheta \beta, \vartheta \gamma)=\vartheta^{2} \int_{-\alpha}^{+\alpha} \int_{-\beta}^{+\beta} \int_{-\gamma}^{+\gamma} \frac{d x d y d z}{r}
$$

$$
\Phi(\vartheta \alpha, \vartheta \beta, \vartheta \gamma)=\vartheta^{2} \Phi(\alpha, \beta, \gamma) \text {. }
$$

Es ist daher $\Phi(\alpha, \beta, \gamma)$ eine homogene Function sweiten Grades von $\alpha, \beta, \gamma$ und genügt also der partiellen Differentialgleichung:

(4.) $2 \Phi=\alpha \frac{\partial \Phi}{\partial \alpha}+\beta \frac{\partial \Phi}{\partial \beta}+\gamma \frac{\partial \Phi}{\partial \gamma}$. 
Hieraus folgt durch Differentiation nach $\alpha$ :

$$
\text { (5.) } \frac{\partial \Phi}{\partial \alpha}=\alpha \frac{\partial^{2} \Phi}{\partial \alpha^{2}}+\beta \frac{\partial^{2} \Phi}{\partial \alpha \partial \beta}+\gamma \frac{\partial^{2} \Phi}{\partial \alpha \partial \gamma},
$$

während die Werthe von $\frac{\partial \Phi}{\partial \beta}$ und $\frac{\partial \Phi}{\partial \gamma}$ aus dem Werthe für $\frac{\partial \Phi}{\partial \alpha}$ durch Vertauschung von $\alpha$ mit $\beta$ oder $\alpha$ mit $\gamma$ erhalten werden.

Nun ist aus der Form von $\Phi(\alpha, \beta, \gamma)$ leicht ersichtlich, dafs :

also ist auch:

$$
\frac{\partial \Phi}{\partial \alpha}=2 \int_{-\beta}^{+\beta} \int_{-\gamma}^{+\gamma} \frac{d y d z}{\sqrt{\alpha^{2}+y^{2}+z^{2}}}
$$

$$
\frac{\partial^{2} \Phi}{\partial \alpha \partial \beta}=4 \int_{-\gamma}^{+\gamma} \frac{d z}{\sqrt{\alpha^{2}+\beta^{2}+z^{2}}}
$$

oder, da sich diese Integration sofort ausführen läfst:

wo:

$$
\text { (6.) } \frac{\partial^{2} \Phi}{\partial \alpha \partial \beta}=4 \log \frac{\varrho+\gamma}{\varrho-\gamma},
$$

Ferner ist:

$$
\rho^{2}=\alpha^{2}+\beta^{2}+\gamma^{2}
$$

$$
\frac{\partial^{2} \Phi}{\partial \alpha^{2}}=-2 \alpha \int_{-\beta}^{+\beta} \int_{-\gamma}^{+\gamma} \frac{d y d z}{\left(\alpha^{2}+y^{2}+z^{2}\right)^{\frac{3}{2}}}
$$

Schreibt man nun für die rechte Seite dieser Gleichung:

und setzt dann:

$$
-\alpha \int_{-\beta}^{+\beta} \int_{-\gamma}^{+\gamma} \frac{d y d\left(z^{2}\right)}{z^{2}\left(\alpha^{2}+y^{2}+z^{2}\right) \frac{\sqrt{\alpha^{2}+y^{2}+z^{2}}}{z}}
$$

wodurch :

$$
\frac{z}{\sqrt{\alpha^{2}+y^{2}+z^{2}}}=u
$$

$$
z^{2}=\frac{\left(\alpha^{2}+y^{2}\right) u^{2}}{1-u^{2}}, \quad \alpha^{2}+y^{2}+z^{2}=\frac{\alpha^{2}+y^{2}}{1-u^{2}}, \quad d\left(z^{2}\right)=\frac{2\left(\alpha^{2}+y^{2}\right) u d u}{\left(1-u^{2}\right)^{2}}
$$

wird, so folgt sofort:

$$
\frac{\partial^{2} \Phi}{\partial \alpha^{2}}=-4 \alpha \gamma \int_{-\beta}^{+\beta} \frac{d y}{\left(\alpha^{2}+y^{2}\right) \sqrt{\alpha^{2}+\gamma^{2}+y^{2}}} .
$$

Dies Integral behandle man auf dieselbe Weise. Es erhält nämlich wiederum leicht die Form:

$$
-2 \alpha \gamma \int_{-\beta}^{+\beta} \frac{d\left(y^{2}\right)}{y^{2}\left(\alpha^{2}+y^{2}\right) \frac{\sqrt{\alpha^{2}+\gamma^{2}+y^{2}}}{y}}
$$


und geht, unbestimmt genommen, durch die Substitution:

wodurch :

$$
\frac{y}{\sqrt{a^{2}+\gamma^{2}+y^{2}}}=z
$$

$$
y^{2}=\frac{\left(\alpha^{2}+\gamma^{2}\right) z^{2}}{1-z^{2}}, \quad \alpha^{2}+y^{2}=\frac{\alpha^{2}+\gamma^{2} z^{2}}{1-z^{2}}, \quad d\left(y^{2}\right)=\frac{2\left(\alpha^{2}+\gamma^{2}\right) z d z}{\left(1-z^{2}\right)^{2}}
$$

wird, über in:

$$
-4 \alpha \gamma \int \frac{d z}{\alpha^{2}+\gamma^{2} z^{2}}
$$

woraus dann leicht geschlossen wird, dafs

$$
\text { (7.) } \frac{\partial^{2} \Phi}{\partial \alpha^{2}}=-8 \operatorname{arctg} \frac{\beta \gamma}{\alpha \rho} ;
$$

$\varrho$ hat die frühere Bedeulung, und der $\operatorname{arctg}$ ist zwischen $-\frac{1}{2} \pi$ und $\frac{1}{2} \pi$ zu nehmen.

Die Gröfsen $\frac{\partial^{2} \Phi}{\partial \beta \partial \gamma}, \frac{\partial^{2} \Phi}{\partial \gamma \partial \alpha} ; \frac{\partial^{2} \Phi}{\partial \beta^{2}}, \frac{\partial^{2} \Phi}{\partial \gamma^{2}}$ erhält man aus (6.) und (7.) durch passende Vertauschung der Gröfsen $\alpha, \beta, \gamma$.

Die Gleichungen (6.) und (7.) enthalten die Lösung der ganzen Aufgabe. Denn es folgt zunächst, indem man aus (6.) -durch Vertauschung von $\beta$ mit $\gamma$ den Werth von $\frac{\partial^{2} \Phi}{\partial \alpha \partial \gamma}$ bildet, und dann die gefundenen Ausdrücke in (5.) einselzt:

$$
\text { (8.) } \frac{\partial \Phi}{\partial \alpha}=4 \beta \log \frac{\rho+\gamma}{\rho-\gamma}+4 \gamma \log \frac{\rho+\beta}{\rho-\beta}-8 \alpha \operatorname{arctg} \frac{\beta \gamma}{\alpha \varrho},
$$

und hieraus und aus den mit Leichtigkeit zu bildenden Werthen von $\frac{\partial \Phi}{\partial \beta}$ und $\frac{\partial \Phi}{\partial \gamma}$ erhält man nach (4.):

$$
\text { (9.) }\left\{\begin{aligned}
\Phi(\alpha, \beta, \gamma)= & 4 \beta \gamma \log \frac{\rho+\alpha}{\rho-\alpha}-4 \alpha^{2} \operatorname{arctg} \frac{\beta \gamma}{\alpha \rho} \\
& +4 \gamma \alpha \log \frac{\rho+\beta}{\rho-\beta}-4 \beta^{2} \operatorname{arctg} \frac{\gamma \alpha}{\beta \rho} \\
& +4 \alpha \beta \log \frac{\rho+\gamma}{\rho-\gamma}-4 \gamma^{2} \operatorname{arctg} \frac{\alpha \beta}{\gamma \rho},
\end{aligned}\right.
$$

wofür auch einfacher geschrieben werden kann:

$$
\Phi(\alpha, \beta, \gamma)=4 S\left[\beta \gamma \log \frac{\rho+\alpha}{\rho-\alpha}-\alpha^{2} \operatorname{arctg} \frac{\beta \gamma}{\alpha \varrho}\right],
$$


Röthig, das Potential eines homogenen rechtwinkligen Parallelepipedums. $2 \dot{53}$

wenn man das Zeichen $S$ so versteht, dafs in dem unter demselben stehenden Ausdrucke die Gröfsen $\alpha, \beta, \gamma$ vertauscht, und die so entstandenen Ausdrücke addirt werden sollen.

Es erhellt hieraus und in Folge der über die arctg gemachten Bemerkung, dafs $\Phi(\alpha, \beta, \gamma)$ mit einem der Argumente sein Zeichen ändert, wie es sein mufs. - Die Gröfse $\varrho$ ist immer positiv zu nehmen.

Ich habe den Ansdruck (9.) zunächst durch Differentiation verificirt. Die Differentiation nach $\alpha$ liefert den in (8.) gegebenen Werth für $\frac{\partial \Phi}{\partial \alpha}$, in dem die übrigen sechs Glieder, welche $\frac{\partial \Phi}{\partial \alpha}$, so wie es aus (9.) folgt, noch enthält, sich gegenseitig vernichten. Daraus folgt dann durch Differentiation nach $\beta$ der in (6.) gegebene Werth von $\frac{\partial^{2} \Phi}{\partial \alpha \partial \beta}$, weil wieder die übrigen Glieder, welche die Differentiation liefert, zusammen identisch Null geben. Hieraus erhält man denn schliefslich

wie es sein mufs.

$$
\text { (10.) } \frac{\partial^{3} \Phi}{\partial \alpha \partial \beta \partial \gamma}=\frac{8}{\rho} \text {, }
$$

Die Gleichung (3.) in Verbindung mit (9.) enthält demnach die Form des Potentiales in Beziehung auf einen beliebigen Punkt und beweist zugleich die Richtigkeit der im Anfange ausgesprochenen Behauptung.

Ist ferner $\boldsymbol{X}(\alpha, \beta, \gamma)$ der Ausdruck auf der rechten Seite von (8.) nach Abscheidung des Factors 4, so hat $\boldsymbol{X}$ die Eigenschaft mit $\beta$ und $\gamma$ das Zeichen zu ändern, aber nicht mit $\alpha$, und da ferner die Ableitung von $\Phi(\alpha, \beta, \gamma)$ nach $\alpha$ hierdurch den Werth $4 \boldsymbol{X}(\alpha, \beta, \gamma)$ erhält, so folgt aus (3.):

$$
\text { (11.) } 2 \frac{\partial \boldsymbol{P}}{\partial \xi}=\Sigma \varepsilon \boldsymbol{X}\left(a+\varepsilon \xi, b+\varepsilon^{\prime} \eta, c+\varepsilon^{\prime \prime} \zeta\right),
$$

und aus (7.)

$$
\text { (12.) } \frac{\partial^{2} \boldsymbol{P}}{\partial \xi^{2}}=-\Sigma \operatorname{arctg} \frac{\left(b+\varepsilon^{\prime} \eta\right)\left(c+\varepsilon^{\prime \prime} \zeta\right)}{(a+\varepsilon \xi)}
$$

wo

$$
\boldsymbol{R}^{2}=(a+\varepsilon \dot{\xi})^{2}+\left(b+\varepsilon^{\prime} \eta\right)^{2}+\left(\boldsymbol{c}+\varepsilon^{\prime \prime} \zeta\right)^{2}
$$

gesetzt ist. Die Gröfsen $\frac{\partial \boldsymbol{P}}{\partial \eta}, \frac{\partial \boldsymbol{P}}{\partial \zeta} ; \frac{\partial^{2} \boldsymbol{P}}{\partial \eta^{2}}, \frac{\partial^{2} \boldsymbol{P}}{\partial \zeta^{2}}$ erhält man aus (11.) und (12.) durch bezügliche Vertauschung der Gröfsen $a, \xi, \varepsilon$, mit $b, \eta$, $\varepsilon^{\prime}$ und $c, \zeta, \varepsilon^{\prime \prime}$. Es sind daher durch die Gleichungen (3.), (11.) und (12.) in VerJournal für Mathematik Bd. LVIII. Heft 3. 


\section{Rïthig, das Potential eines homogenen rechtwinkligen Parallelepipedums.}

bindung mit (8.) und (9.) sämmtliche auf die Anziehung eines homogenen rechtwinkligen Parallelepipedums bezügliche Gröfsen gefunden.

Obgleich nun die vorstehende Herleitung für das Potential eines rechtwinkligen Parallelepipedums nichts zu wünschen übrig läfst, so möchte es doch von Interesse sein, zu zeigen, dafs der gefundene Ausdruck auch auf ziemlich einfache Weise nach der Methode verificirt werden kann, welche Dirichlet im $32^{\text {sten }}$ Bande dieses Journals gegeben hat. Dirichlet beweist dort, dafs ein Ausdruck $\boldsymbol{P}$ dann die richtige Form des Potentiales enthält, wenn:

1) $\boldsymbol{P}$ und seine ersten partiellen Differentialquotienten endliche und continuirliche Functionen von $\xi, \eta, \zeta$ innerhalb des ganzen Raumes sind,

2) die Ausdrücke $\boldsymbol{\xi} \boldsymbol{P}, \eta \boldsymbol{P}, \zeta \boldsymbol{P} ; \boldsymbol{\xi}^{2} \frac{\partial \boldsymbol{P}}{\partial \xi}, \eta^{2} \frac{\partial \boldsymbol{P}}{\partial \eta}, \zeta^{2} \frac{\partial \boldsymbol{P}}{\partial \zeta}$ im ganzen Raume endliche Werthe nicht überschreiten,

3) die Gröfsen $\frac{\partial^{2} \boldsymbol{P}}{\partial \dot{\xi}^{2}}, \frac{\partial^{2} \boldsymbol{P}}{\partial \eta^{2}}, \frac{\partial^{2} \boldsymbol{P}}{\partial \zeta^{2}}$ im ganzen Raume endlich und eindeutig sind und der Differentialgleichung

$$
\Delta \boldsymbol{P}=\frac{\partial^{2} P}{\partial \xi^{2}}+\frac{\partial^{2} P}{\partial \eta^{2}}+\frac{\partial^{2} P}{\partial \zeta^{2}}=-4 \pi \varkappa
$$

genügen, wo für einen inneren Punkt $(\xi, \eta, \zeta) x=1$ zu setzen ist, für einen äufseren $x=0$.

Zu der dritten Bedingung ist zu bemerken, dafs sie für den hier vorliegenden Fall ausgesprochen ist, nämlich für einen homogenen Körper, dessen Dichtigkeit gleich der Einheit gesetzt ist.

Es soll nun gezeigt werden, dafs der für das Potential gegebene Ausdruck diesen drei Bedingungen genügt.

Zunächst ist klar, dafs die in (3.) und (11.) aufgestellten Formen des Potentiales und seiner ersten Ableitungen die erste Bedingung erfüllen. Denn die aus (8.) und (9.) ersichtliche Bildung von $\Phi$ und $\boldsymbol{X}$ zeigt, dafs das Potential und seine ersten Ableitungen auf stetige Weise aus Functionen zusammengesetzt sind, die für alle Werthe dẹ Argumente zwischen $-\infty$ und $+\infty$ stetig bleiben.

Was die zweite Bedingung betrifft, so ist klar, dafs sie erfült ist, so lange $\xi, \eta, \zeta$ endlich bleiben. Denn die Gleichungen (3.) und (11.) in Verbindung mit (8.) und (9.) zeigen, dafs alle Glieder, aus denen das Potential und seine ersten Ableitungen zusammengeselzt sind, endlich bleiben, so lange 
$\xi, \eta, \zeta$ endliche Werthe haben. Es bleibt daher nur noch zu zeigen, dafs diese Bedingung auch erfüllt ist, wenn $(\xi, \eta, \zeta)$ im Unendlichen liegt. Um die grofsen Weitläufigkeiten zu ersparen, welche eine directe Behandlung der Gleichungen (3.) und (11.) für diesen Fall mit sich führt, sollen jelzt die Formen des Polentiales und seiner crsten Ableitungen in zweckdienlicher Weise umgeformt werden.

Da $\Phi(\alpha, \beta, \gamma)$ mit $\alpha$ sein Zeichen ändert, so darf für (3.) geschrieben werden:

$$
8 \boldsymbol{P}=\Sigma \varepsilon \Phi\left(\xi+\varepsilon \boldsymbol{a}, \boldsymbol{b}+\varepsilon^{\prime} \eta, \boldsymbol{c}+\varepsilon^{\prime \prime} \zeta\right) .
$$

Läfst man nun der Abkürzung wegen zunächst die beiden letzten Argumente in $\Phi$ weg, so ist klar, dafs die rechts stehende Summe identisch ist, mit der folgenden :

$$
\Sigma \varepsilon[\boldsymbol{\Phi}(\xi+\varepsilon \boldsymbol{a})-\boldsymbol{\Phi}(\xi)]
$$

und da nach dem Taylorschen Satze:

$$
\begin{gathered}
\boldsymbol{\Phi}(\xi+\varepsilon \boldsymbol{u})-\boldsymbol{\Phi}(\xi)=\varepsilon \boldsymbol{a} \partial_{\xi} \boldsymbol{\Phi}(\xi+\boldsymbol{\vartheta} \boldsymbol{\varepsilon} \boldsymbol{a}), \\
\boldsymbol{0}<\boldsymbol{\vartheta}<1,
\end{gathered}
$$

wo $\partial_{\xi} \Phi$ die erste Ableilung nach $\xi$ bedeutet,

so folgt:

$$
8 \boldsymbol{P}=a \Sigma \partial_{\xi} \Phi\left(\xi+\vartheta \varepsilon a, b+\varepsilon^{\prime} \eta, c+\varepsilon^{\prime \prime} \zeta\right)
$$

Nun ändert aber, wie oben bemerkt worden, $\boldsymbol{X}(\alpha, \beta, \gamma)$ mit $\beta$ sein Zeichen, also auch $\partial_{\xi} \Phi$ mit $b+\varepsilon^{\prime} \eta$; es darf daher, wie oben, für die vorstehende Gleichung geschrieben werden:

$$
8 \boldsymbol{P}=a \Sigma \varepsilon^{\prime} \partial_{\xi} \Phi\left(\xi+\vartheta \varepsilon a, \eta+\varepsilon^{\prime} b, c+\varepsilon^{\prime \prime} \zeta\right),
$$

oder, indem man wie vorher weiter schliefst:

$$
8 \boldsymbol{P}=a b \Sigma \partial_{\xi, \eta}^{2} \Phi\left(\xi+\vartheta \varepsilon a, \eta+\vartheta^{\prime} \varepsilon^{\prime} b, c+\varepsilon^{\prime \prime} \zeta\right),
$$

wo $\partial_{\xi, \eta}^{2}$ die zweite Ableitung nach $\xi$ und $\eta$ bedeutet, und $0<\vartheta^{\prime}<1$. Endlich ändert auch $\partial_{\alpha, \beta}^{2} \Phi(\alpha, \beta, \gamma)$ mit $\gamma$ sein Zeichen, wie aus (6.) folgt, also erhält man durch Wiederholung desselben Verfahrens:

$$
8 \boldsymbol{P}=a b c \Sigma \partial_{\xi, \eta, \zeta}^{3} \Phi\left(\xi+\vartheta \varepsilon \|, \eta+\vartheta^{\prime} \varepsilon^{\prime} b, \zeta+\vartheta^{\prime \prime} \varepsilon^{\prime \prime} c\right),
$$

wo $\partial_{\xi, \eta, \zeta}^{3}$ die dritte Ableitung nach $\xi$ und $\eta$ und $\zeta$ bedeutet und $0<\vartheta^{\prime \prime}<1$. Hieraus folgt aber nach (10.):

$$
\text { (13.) } \quad \boldsymbol{P}=\operatorname{abc} \Sigma \frac{1}{R_{1}},
$$


wo

$$
\boldsymbol{R}_{\mathbf{1}}^{2}=(\boldsymbol{\xi}+\boldsymbol{\vartheta} \boldsymbol{\varepsilon} \boldsymbol{a})^{2}+\left(\eta+\boldsymbol{\vartheta}^{\prime} \boldsymbol{\varepsilon}^{\prime} \boldsymbol{b}\right)^{2}+\left(\boldsymbol{\zeta}+\boldsymbol{\vartheta}^{\prime \prime} \varepsilon^{\prime \prime} \boldsymbol{c}\right)^{2}
$$

gesetzt worden, und die Gröfsen $\vartheta, \vartheta^{\prime}, \vartheta^{\prime \prime}$ zwischen 0 und 1 liegen.

Zu dieser Gleichung ist zu bemerken, dafs die Gröfsen $\boldsymbol{\vartheta}, \boldsymbol{\vartheta}^{\prime}, \boldsymbol{\vartheta}^{\prime \prime}$ eigentlich mit Indices versehen sein müssen, welche anzeigen, dafs sie für die verschiedenen Werthe von $\varepsilon, \varepsilon^{\prime}, \varepsilon^{\prime \prime}$ nicht dieselben Werthe besitzen. Diese Unterscheidung ist jedoch weggelassen worden, weil keine andere Eigenschaft der Gröfsen $\vartheta, \vartheta^{\prime}, \vartheta^{\prime \prime}$ gebraucht wird, als die, dafs sie zwischen 0 und 1 liegen. Ferner ist ersichtlich, dafs man Ausdrücke für die ersten Ableitungen nach $\xi, \eta, \zeta$ aus (13.) durch Differentiation nach $\xi, \eta, \zeta$ unter der Voraussetzung, dafs $\vartheta, \vartheta^{\prime}, \vartheta^{\prime \prime}$ constant sind, ableiten darf. Denn würde man die eigentlichen Ausdrücke für die Ableitungen, so wie sie aus der Gleichung (11.) folgen, in derselben Weise behandeln, wie es mit der Form des Polentiales selbst geschehen ist, wobei man bemerken möge, dafs dies möglich ist, weil $\boldsymbol{X}(\alpha, \beta, \gamma)$ denselben Werth behält, wenn $\alpha$ sein Zeichen ändert, also $\boldsymbol{X}(\xi+\varepsilon a)$ für $\boldsymbol{X}(a+\varepsilon \xi)$ geschrieben werden darf, so würde man gerade auf die Ausdrücke kommen, welche man durch Differentiation unter der Voraussetzung, dafs $\vartheta, \vartheta^{\prime}, \vartheta^{\prime \prime}$ constant sind, aus der Gleichung (13.) direct erhält. Natürlich sind dann die Gröfsen $\boldsymbol{\vartheta}, \boldsymbol{\vartheta}^{\prime}, \boldsymbol{\vartheta}^{\prime \prime}$ in den Ableitungen mit denen der Gleichung (13.) nicht identisch, aber diese Eigenschaft wird auch nicht angewendet.

Nach diesen Bemerkungen folgt nun aus (13.):

$\frac{\partial \boldsymbol{P}}{\partial \xi}=-a b c \Sigma \frac{\xi+\vartheta_{\varepsilon} a}{\boldsymbol{R}_{1}^{3}}, \quad \frac{\partial \boldsymbol{P}}{\partial \eta}=-\boldsymbol{a b c} \Sigma \frac{\eta+\boldsymbol{\vartheta}^{\prime} \varepsilon^{\prime} b}{\boldsymbol{R}_{1}^{3}}, \frac{\partial \boldsymbol{P}}{\partial \zeta}=-a b c \Sigma \frac{\zeta+\boldsymbol{\vartheta}^{\prime \prime} \varepsilon^{\prime \prime} c}{\boldsymbol{R}_{1}^{3}}$, und diese Gleichungen in Verbindung mit (13.) zeigen ohne Weiteres; dafs die Ausdrücke $\boldsymbol{\xi} \boldsymbol{P}, \eta \boldsymbol{P}, \zeta \boldsymbol{P} ; \boldsymbol{\xi}^{2} \frac{\partial \boldsymbol{P}}{\partial \xi}, \eta^{2} \frac{\partial \boldsymbol{P}}{\partial \eta}, \zeta^{2} \frac{\partial \boldsymbol{P}}{\partial \zeta}$ immer endlich bleiben, wie viele von den Gröfsen $\xi, \eta, \zeta$ auch unendlich werden, und welche Werthe die Verhältnisse $\eta_{l}: \xi$, und $\zeta: \xi$ auch im Unendlichen haben mögen. Der zweiten Bedingung wird daher vollständig genügt.

Auch die dritle Bedingung ist erfüllt. Denn aus der Gleichung (12.) folgt, dafs die Gröfsen $\frac{\partial^{2} P}{\partial \xi^{2}}, \frac{\partial^{2} P}{\partial \eta^{2}}, \frac{\partial^{2} P}{\partial \zeta^{2}}$ eindeutig sind, da die in ihnen vorkommenden arcig immer zwischen $-\frac{1}{2} \pi$ und $\frac{1}{2} \pi$ liegen, und ferner, dafs diese Werthe überall endlich bleiben, selbst wenn $(\xi, \eta, \zeta)$ im Unendlichen liegt. Es ist daher nur noch zu zeigen, dafs sie der gegebenen partiellen Differentialgleichung $\triangle \boldsymbol{P}=-4 \pi \%$ genügen. 
Röthig, das Potential eines homogenen rechtwinkligen Parallelepipedums. 257

Hierzu ist nach (7.)

also auch

$$
\frac{\partial^{2} \Phi}{\partial \alpha^{2}}=-8 \operatorname{arctg} \frac{\beta \gamma}{\alpha \rho}
$$

$$
\frac{\partial^{2} \Phi}{\partial \beta^{2}}=-8 \operatorname{arctg} \frac{\gamma \alpha}{\beta \varrho}
$$

und

$$
\frac{\partial^{2} \Phi}{\partial \gamma^{2}}=-8 \operatorname{arctg} \frac{\alpha \beta}{\gamma \varrho} .
$$

Durch Addition dieser Ausdrücke erhält man:

$$
\text { (14.) } \Delta \Phi=-4 \pi \text {, }
$$

wenn die Gröfsen $\alpha, \beta, \gamma$ positiv sind. Denn die beiden ersten arctg geben addirt $\operatorname{arctg} \frac{\gamma \varrho}{\alpha \beta}$, und dieser mit dem letzten zusammen giebt, wie man sofort sieht, $\frac{1}{2} \pi$.

Ist nun $(\xi, \eta, \zeta)$ ein innerer Punkt, so sind die Argumente sämmtlicher $\Phi$ in (3.) positiv und man erhält daher sofort mit Hülfe von (14.)

$$
\triangle \boldsymbol{P}=-4 \pi \text {. }
$$

Ist dagegen $(\xi, \eta, \zeta)$ ein äufserer Punkt, so ist mindestens eine der Bedingungen $\xi>a, \eta>b, \zeta>c$ erfüllt. Sei z. B. $\xi>a$, so darf für (3.) geschrieben werden:

$$
8 \boldsymbol{P}=\Sigma \varepsilon \boldsymbol{\Phi}\left(\xi+\varepsilon a, \eta+\varepsilon^{\prime} b, \zeta+\varepsilon^{\prime \prime} c\right),
$$

und da nun in allen $\Phi$ das erste Argument jedenfalls positiv ist, so erhält man durch (14.)

$$
\Delta \boldsymbol{P}=-\frac{1}{2} \pi \Sigma \lambda \cdot \varepsilon,
$$

wo $\lambda$ einen von dem Verhalten der Gröfsen $\eta$, $\zeta$, also jedenfalls nur von den Zeichen $\varepsilon^{\prime}$ und $\varepsilon^{\prime \prime}$ abhängigen Coefficienten bedeutet. Da also in der vorstehenden Gleichung die Summe nach $\varepsilon$ verschwindet, so ist

$$
\Delta \boldsymbol{P}=\mathbf{0}
$$

und dasselbe tritt ein, wenn man von einer der Bedingungen $\eta>b, \zeta>c$ ausgeht, weil dann jedenfalls die Summen nach $\varepsilon^{\prime}$ oder $\varepsilon^{\prime \prime}$ verschwinden. Es ist daher für jeden äufseren Punkt

$$
\Delta \boldsymbol{P}=\mathbf{0}
$$

und damit gezeigt, dafs auch der dritten Bedingung genügt wird.

Hiermit sind also die gegebenen Ausdrücke nach der Dirichletschen Methode vollständig verificirt, und es folgt daher auch auf diese Weise, dafs 
258 Röthig, das Potential eines homogenen rechtwinkligen Parallelepipedums.

(3.) das Potential eines homogenen rechtwinkligen Parallelepipedums darstell. - Durch die Kenntnifs des Raumpolentiales wird man nun auch in den Stand gesetzt für alle Lagen von $(\xi, \eta, \zeta)$ das Flächenpotential anzugeben. Denn es leuchtet sofort ein, dafs das Potential der Oberfläche eines rechtwinkligen Parallelepipedums mit den Seiten $2 a, 2 b, 2 c$ in Beziehung anf einen Punkt $(\xi, \eta, \zeta)$, welches mit $\boldsymbol{V}(a, b, c, \xi, \eta, \zeta)$ bezeichnet werden möge, gegeben ist durch die Gleichung:

$$
\boldsymbol{V}=\frac{\partial \boldsymbol{P}}{\partial \boldsymbol{a}}+\frac{\partial \boldsymbol{P}}{\partial \iota}+\frac{\partial \boldsymbol{P}}{\partial \boldsymbol{c}},
$$

wo $\boldsymbol{P}$ das entsprechende Raumpotential bedeutet. - Ich bemerke endlich noch zum Schlusse, dafs dieselbe Methode auch ausreicht, um das Potential eines beliebigen homogenen Parallelepipedums zu finden.

Berlin, im Juni 1860. 